\title{
Study of Backscattering Effects on Particle Identification
}

\section{J. Wu ${ }^{a}$ and E. S. Seo ${ }^{a, b}{ }^{*}$ on behalf of the ISS-CREAM Collaboration}

(a complete list of authors can be found at the end of the proceedings)

aInstitute for Physical Science and Technology, University of Maryland,

College Park, MD, USA

bDepartment of Physics, University of Maryland,

College Park, MD, USA

E-mail: Jayoungeumd.edu, seo@umd.edu

One of the consequences of having a high-density calorimeter as part of an experiment is a large number of secondary shower particles generated in the calorimeter -- some of which scatter back up towards the charge measurement devices. This so-called "backscatter effect" can interfere severely with accurate charge measurement of the primary nucleus, especially at high energies, as the number of backscattered particles increases with the incident energy. In this analysis, we study the effect of backscattered particles on particle identification by simulating the ISS-CREAM instrument detector model response using the GEANT3 simulation package [1] with the FLUKA hadronic model [2]. Our study shows the importance of the fine segmentation of the charge detectors above the calorimeter. This segmentation minimizes backscattered particle contamination in the same charge detector segment as the incident particle, which helps avoid charge misidentification. Here we present simulation results regarding charge measurements, including tracking resolution, backscattering effects, and charge determination efficiency.

37th International Cosmic Ray Conference (ICRC 2021)

July 12th-23rd, 2021

Online-Berlin, Germany

\section{*Presenter}

(C) Copyright owned by the author(s) under the terms of the Creative Commons 


\section{Introduction}

When a high-energy proton (or nucleus) enters the calorimeter module, it soon undergoes a nuclear interaction, generating a large number of secondary particles (mainly pions) that carry off a significant fraction of its energy. The neutral pions decay almost immediately into photon pairs and initiate electromagnetic (EM) showers or cascades (extensive collections of electrons, positrons, and photons). The surviving primary particle and charged secondaries then continue into the calorimeter. Some of these energetic charged hadrons will interact again, and fractions of their energy are also transferred to EM cascades.

Most high-energy shower particles have a 'forward' momentum component relative to the incident trajectory. However, many lower-energy particles move 'backward'. Signals from some of these particles will overlay that of the primary particle in the charge detector, making charge measurement more challenging. Indeed, this is a rather widely accepted explanation for the misidentification of protons as $\mathrm{He}$ nuclei in past experiments [3]. To study the effect of backscattered particles on particle identification, we have developed a simulation model for the ISS-CREAM instrument detector response using the Monte Carlo method.

\section{ISS-CREAM Instrument}

The ISS-CREAM instrument consists of complementary and redundant particle detectors (Figure 1). An ionization calorimeter determines the energy of cosmic ray particles, provides tracking, and a high energy trigger. Carbon-Targets induce hadronic interactions. The four-layer Silicon charge detectors (SCD) provide precise charge measurements. Top/bottom counting detectors $(\mathrm{TCD} / \mathrm{BCD})$ provide shower profiles for electron/hadron separation and a lower energy trigger. The boronated scintillator detector (BSD) provides additional electron/hadron discrimination using thermal neutrons produced by particles that interact within the calorimeter. For a detailed description of ISS-CREAM instrument configuration, refer to [5].

\section{Monte Carlo Simulations}

Our simulation model is based on the GEANT3 Monte Carlo simulation toolkit, written in Fortran, and has a large set of physics processes handling the complicated interactions of particles in matter. GEANT4 is the successor of GEANT3, re-designed for the next generation of experiments using an object-oriented environment, and is widely used for particle simulations. However, for this study, we used GEANT3 for consistency with the CREAM balloon experiment that had used GEANT3. Calorimeter science in GEANT4 is unchanged. We have tested several hadron interaction packages and chosen FLUKA as the baseline for our simulation model [6]. The ISS-CREAM instrument [7] simulation was configured to be as realistic as possible [4]. In this study, protons were generated isotropically over an incident energy range from $100 \mathrm{GeV}$ to 500 $\mathrm{TeV}$. A typical event passes first through the charge module, then interacts in the carbon target, and finally develops a shower in the calorimeter (Figure 2).

\section{Data Analysis}

The simulation results presented here were obtained by selecting events in which the particles enter the SCD, pass through all the calorimeter layers, have their first interaction anywhere in the carbon target, and deposit significant amounts of energy in many $(\geq 6)$ layers of the calorimeter for tracking. A sparsification scheme (discarding channels without signal 
significantly above the pedestal) is implemented with a $3 \mathrm{MeV}$ threshold that does not degrade calorimeter performance over the incident energy range.

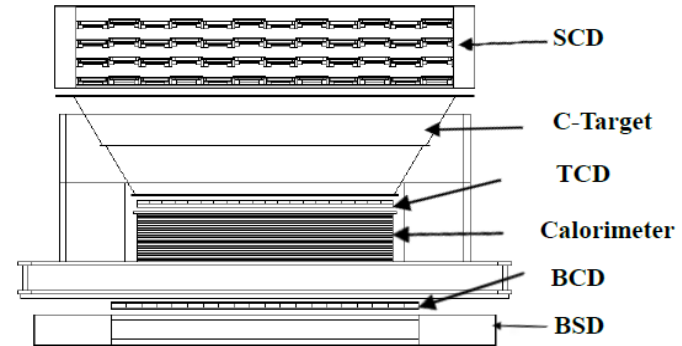

Figure 1. Simulated ISS-CREAM detector configuration [4]

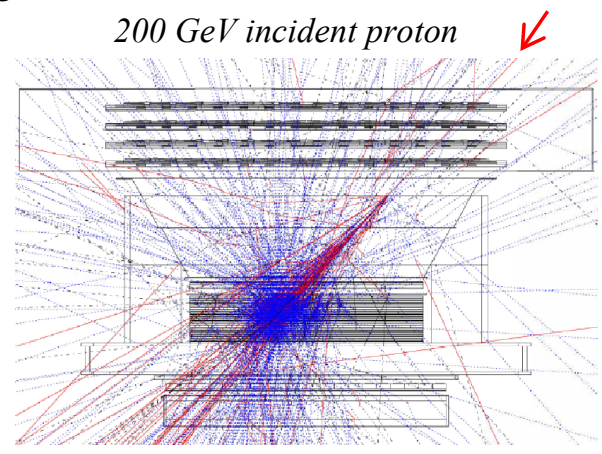

Figure 2. An example of a simulated event: gamma (blue), charged particles excluding muons (red), and neutral hadrons or neutrinos (black) [4]

\subsection{Event Reconstruction}

A tracking algorithm is used to reconstruct the incident particle trajectory by calculating the energy deposit centroid in each calorimeter layer, thereby providing up to $10 \times 10(\mathrm{x}, \mathrm{y})$ cascade coordinate pairs. The cascade axis is determined by the linear fitting of these coordinates separately in the $\mathrm{x}$ and $\mathrm{y}$ directions. Two requirements are applied to the tracking algorithm to reduce fluctuations: (1) each calorimeter layer used in the fitting is required to have an energy deposit greater than $3 \%$ of the total calorimeter energy deposit, (2) an event is accepted if it has at least three calorimeter layers that satisfy the above condition in both the $\mathrm{x}$ and $\mathrm{y}$ direction -otherwise, it is rejected.

\subsection{Trajectory Resolution}

The deviation between the actual incident position and the reconstructed position is a Gaussian distribution and the one $\sigma$ error (position resolution) at the top of the calorimeter is $\sim 0.18 \mathrm{~cm}$ as shown in Figure 3: for the $\mathrm{x}$ direction (left), and the y direction (right).
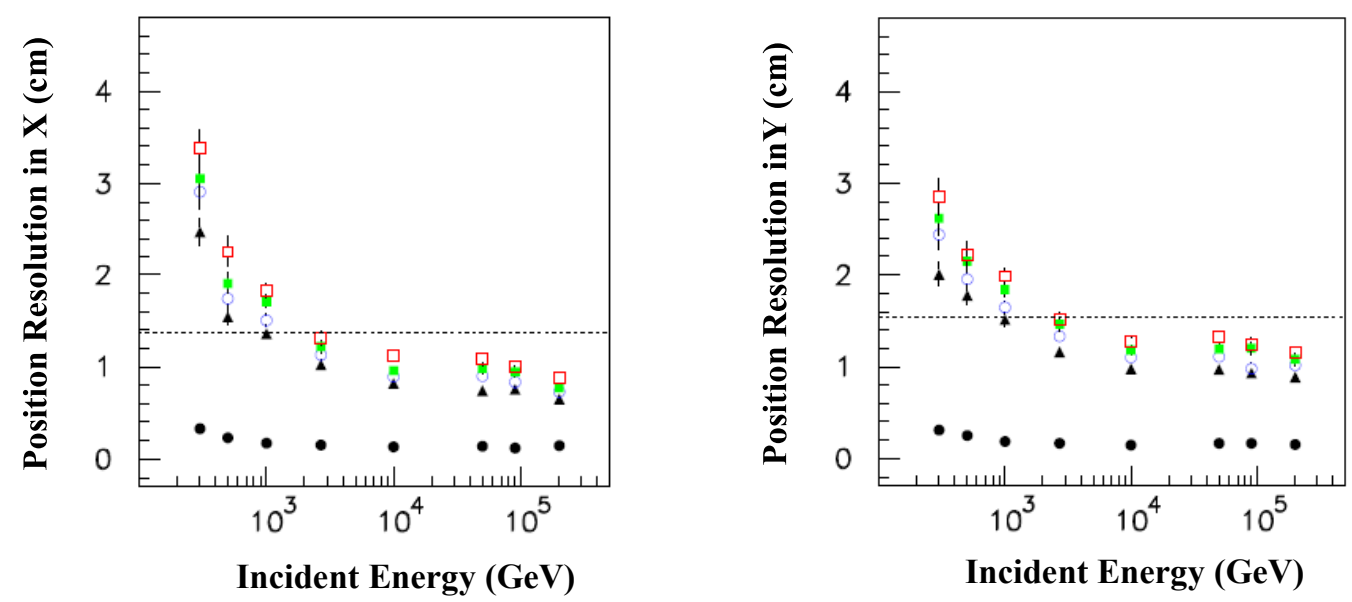

Figure 3. Incident energy dependence of the position resolution for incident protons; calorimeter (filled black circles), distance from top of the calorimeter $38.45 \mathrm{~cm}$ (open red squares), $34.65 \mathrm{~cm}$ (filled green squares), $30.85 \mathrm{~cm}$ (open blue circles), $27.05 \mathrm{~cm}$ (filled black triangles) from top to bottom. 
By extrapolating the reconstructed trajectory to the 4 layers of SCD, the entrance position of the primary particle at each SCD layer is calculated. The distance from the top of calorimeter to each SCD layer is $38.45 \mathrm{~cm}, 34.65 \mathrm{~cm}, 30.85 \mathrm{~cm}$, and $27.05 \mathrm{~cm}$, from top to bottom. The position resolution at each SCD layer gets worse with distance from the calorimeter due to the increasing lever arm. The position resolution at the SCD is about 1 pixel $(\mathrm{E} \geq 2.7 \mathrm{TeV})$. Dotted lines on Figure 3 represent the SCD pixel width (left) and length (right). The SCD resolution is not good at low energies $(\mathrm{E}<2.7 \mathrm{TeV})$ due to undeveloped shower cores.

\subsection{Charge Determination Algorithm}

In order to determine the incident particle charge, the reconstructed shower axis from the calorimeter is extrapolated to the SCD and a $10 \mathrm{~cm} \times 10 \mathrm{~cm}$, circle of confusion, centered on the extrapolated position, was scanned to select the highest signal pixel [8]. If no signal is found, the event is considered not to satisfy the charge requirement and is rejected. If found, the highest signal is then corrected for the particle path length (calculated from the reconstructed incidence angle). The signal reflects the ionization energy loss per unit path length $(\mathrm{dE} / \mathrm{dx})$ of an incident particle in the $\mathrm{SCD}$, which is proportional to $\mathrm{Z}^{2}$. The resulting $\mathrm{Z}$ distribution is shown in Figure 4. Events with $Z \leq 1.732$ are identified as protons. Their peak values are 1 . However, as energy increases, both the mean and RMS values get larger due to more backscatter at higher energies. The mean also increases due to being closer to the calorimeter.
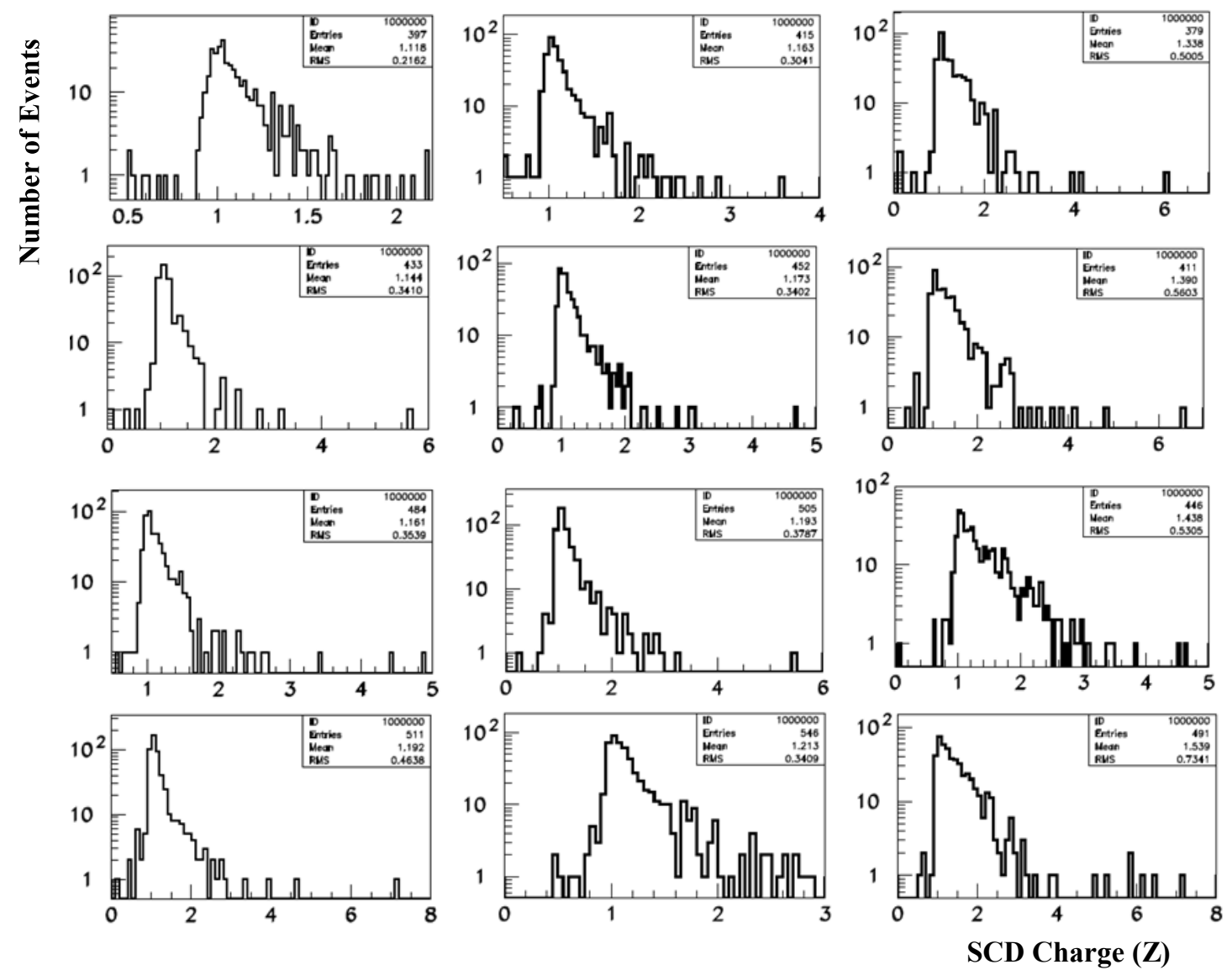

Figure 4: SCD layer charge signal distributions for protons with incident energies of $3 \mathrm{TeV}, 10 \mathrm{TeV}$, and 200 $\mathrm{TeV}$ from left to right (for the four different layer distances of $38.45 \mathrm{~cm}, 34.65 \mathrm{~cm}, 30.85 \mathrm{~cm}$, and $27.05 \mathrm{~cm}$ from top to bottom) 


\subsection{Backscattering Effect versus SCD Distance from the Calorimeter}

Figure 5 shows the charge determination efficiency: the fraction of events correctly identified as a proton among the reconstructed proton MC events that traverse SCD active area. The efficiencies at low energies are different from those at high energies. At lower energies $(\mathrm{E}<$ $2.7 \mathrm{TeV}$ ) the charge determination efficiency increases with energy mainly due to tracking uncertainty: Since shower core is not well-developed at low energies, the tracking resolution gets better as energy increases. However, at higher energies $(\mathrm{E}>30 \mathrm{TeV})$, the efficiency decreases as energy increases due to the presence of more backscattered particles. In the low energy range, the charge determination efficiency is mainly affected by the tracking resolution, while in the high energy range it is mainly affected by backscattered particles. At mid-range energies $(2.7 \mathrm{TeV} \leq \mathrm{E}$ $\leq 30 \mathrm{TeV}$ ), the efficiency is about $90 \%$-- relatively independent of the incident energy.

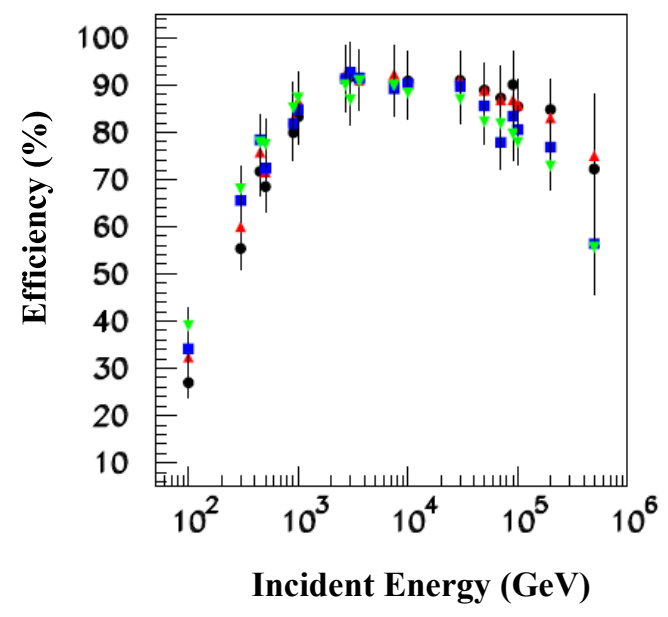

Figure 5: Incident energy dependence of charge determination efficiency: distance from top of the calorimeter is $27.05 \mathrm{~cm}$ (green upside down triangle), $30.85 \mathrm{~cm}$ (blue rectangle), $34.65 \mathrm{~cm}$ (red triangle), and $38.45 \mathrm{~cm}$ (black circle)

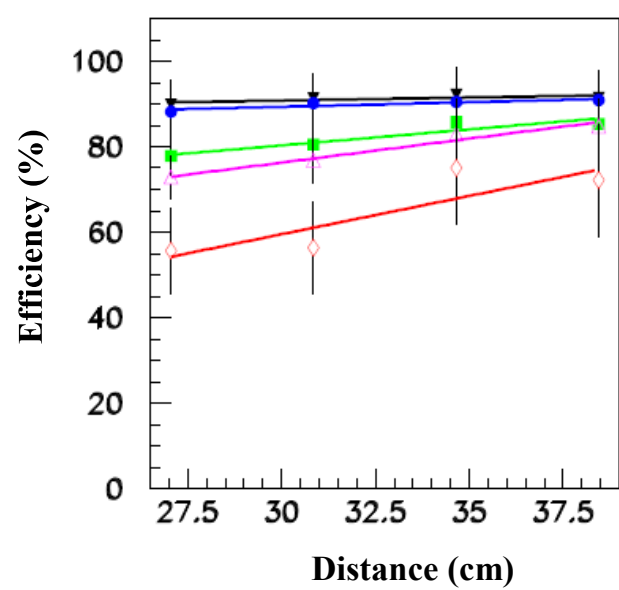

Figure 6: SCD layer distance dependence of charge determination efficiency: incident energy $2.7 \mathrm{TeV}$ (black upside down triangle), $10 \mathrm{TeV}$ (blue circle), $100 \mathrm{TeV}$ (green rectangle), $200 \mathrm{TeV}$ (pink triangle), $500 \mathrm{TeV}$ (red diamond), and linear fit (solid line)

The SCD layer distance dependence of the charge determination efficiency is shown as a function of distance from top of the calorimeter in Figure 6. It can be seen that the charge efficiency is quite linear with distance. The efficiency is improved with distance from the calorimeter due to the reduction of backscattered particles. At $\mathrm{E}=200 \mathrm{TeV}$ the efficiency is $\sim 86 \%$ at $38.45 \mathrm{~cm}$ while it is $\sim 73 \%$ at $27.05 \mathrm{~cm}$. The slope steepens as energy increases. Table 1 is a summary of the linear fit.

Figure 7 shows the misidentified proton $(Z>1.732)$ fraction among the reconstructed $M C$ events thrown with $Z>0$, with respect to incident energy. At up to $\sim 3 \mathrm{TeV}$ incident energy the misidentified fraction is $\sim 2.75 \%$, and the trend is almost flat. However, over the higher energy ranges the misidentified fraction increases with energy due to the increase of backscattered secondary particles. The log of the fraction in \% is proportional (slope of $0.29 \pm 0.02$ ) to the log of the incident energy. Figure 8 plots the misidentified fraction as a function of distance from top of the calorimeter. The misidentified fraction linearly decreases as distance increases and the slope is steeper as incident energy increases. Table 2 is a summary of the linear fits. At $1 \mathrm{TeV}$ the 
misidentified fraction decreases by $0.15 \%$ for each $1 \mathrm{~cm}$ from the top of the calorimeter while at $500 \mathrm{TeV}$ by $1.97 \%$. The misidentified fraction is $2.28 \%$ with $1 \mathrm{TeV}$ (at $38.45 \mathrm{~cm}$ ) and $41.6 \%$ with $500 \mathrm{TeV}$ (at $27.05 \mathrm{~cm}$ ).

\begin{tabular}{|l|ll|llll|}
\hline $\begin{array}{l}\text { incident } \\
\text { energy }\end{array}$ & intercept & slope & $\begin{array}{l}\mathbf{2 7 . 0 5} \\
\text { (cm) }\end{array}$ & $\begin{array}{l}\mathbf{3 0 . 8 5} \\
\text { (cm) }\end{array}$ & $\begin{array}{l}\mathbf{3 4 . 6 5} \\
\text { (cm) }\end{array}$ & $\begin{array}{l}\mathbf{3 8 . 4 5} \\
\text { (cm) }\end{array}$ \\
\hline $2.7 \mathrm{TeV}$ & 86.41 & $0.15 \pm 0.71$ & $90.37 \%$ & $90.93 \%$ & $91.49 \%$ & $92.04 \%$ \\
$10 \mathrm{TeV}$ & 83.00 & $0.21 \pm 0.69$ & $88.74 \%$ & $89.55 \%$ & $90.35 \%$ & $91.16 \%$ \\
$100 \mathrm{TeV}$ & 57.92 & $0.75 \pm 0.60$ & $78.13 \%$ & $80.97 \%$ & $83.80 \%$ & $86.64 \%$ \\
$200 \mathrm{TeV}$ & 42.67 & $1.12 \pm 0.67$ & $73.00 \%$ & $77.26 \%$ & $81.52 \%$ & $85.78 \%$ \\
$500 \mathrm{TeV}$ & 5.87 & $1.79 \pm 1.35$ & $54.27 \%$ & $61.07 \%$ & $67.87 \%$ & $74.67 \%$ \\
\hline
\end{tabular}

Table 1: Linear fit of charge determination efficiency over distance from top of calorimeter: incident energies of $2.7 \mathrm{TeV}, 10 \mathrm{TeV}, 100 \mathrm{TeV}, 200 \mathrm{TeV}$ and $500 \mathrm{TeV}$.

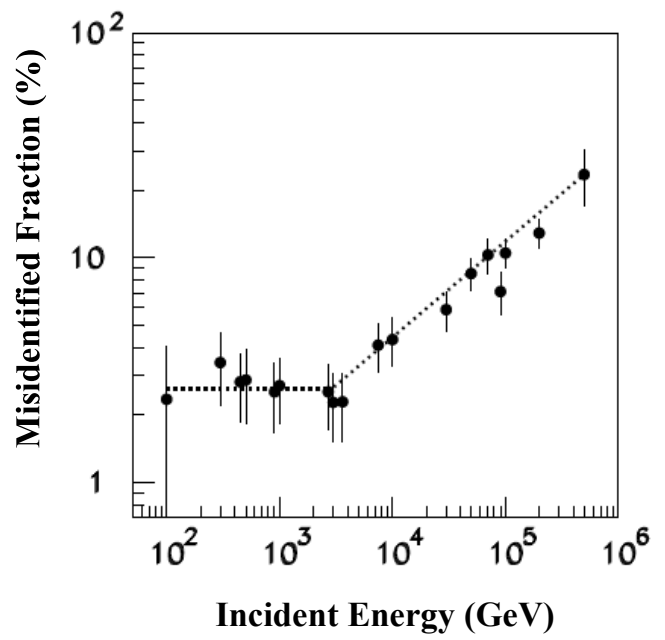

Figure 7: Incident energy dependence of the misidentified fraction at $38.45 \mathrm{~cm}$ from the top of the calorimeter. The dotted line is a guide to the eye.

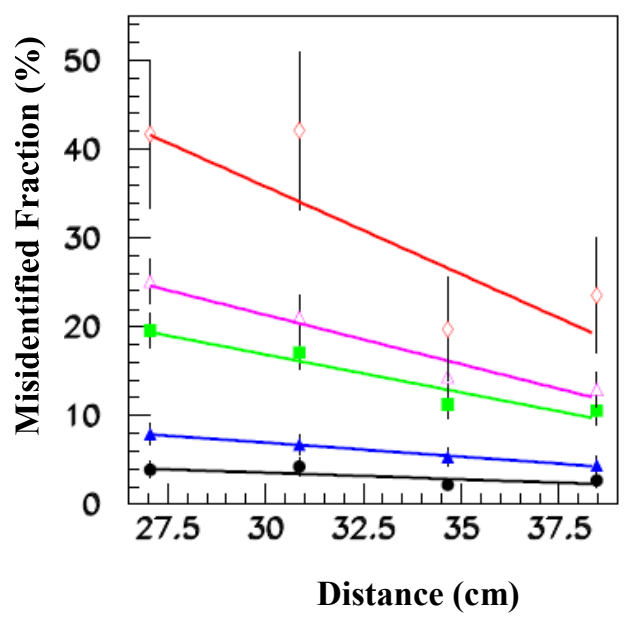

Figure 8: SCD layer distance dependence of the misidentified fraction: incident energy 1 $\mathrm{TeV}$ (black circle), $10 \mathrm{TeV}$ (blue triangle), $100 \mathrm{TeV}$ (green rectangle), $200 \mathrm{TeV}$ (pink triangle), $500 \mathrm{TeV}$ (red diamond), and linear fit (solid line).

\begin{tabular}{|l|ll|llll|}
\hline $\begin{array}{l}\text { incident } \\
\text { energy }\end{array}$ & intercept & slope & $\begin{array}{l}\mathbf{2 7 . 0 5} \\
\text { (cm) }\end{array}$ & $\begin{array}{l}\mathbf{3 0 . 8 5} \\
\text { (cm) }\end{array}$ & $\begin{array}{l}\mathbf{3 4 . 6 5} \\
\text { (cm) }\end{array}$ & $\begin{array}{l}\mathbf{3 8 . 4 5} \\
\text { (cm) }\end{array}$ \\
\hline $1 \mathrm{TeV}$ & 8.04 & $-0.15 \pm 0.10$ & $3.99 \%$ & $3.42 \%$ & $2.85 \%$ & $2.28 \%$ \\
$10 \mathrm{TeV}$ & 16.44 & $-0.32 \pm 0.13$ & $7.87 \%$ & $6.67 \%$ & $5.46 \%$ & $4.26 \%$ \\
$100 \mathrm{TeV}$ & 42.48 & $-0.85 \pm 0.21$ & $19.38 \%$ & $16.13 \%$ & $12.89 \%$ & $9.64 \%$ \\
$200 \mathrm{TeV}$ & 54.69 & $-1.11 \pm 0.26$ & $24.61 \%$ & $20.38 \%$ & $16.16 \%$ & $11.93 \%$ \\
$500 \mathrm{TeV}$ & 95.02 & $-1.97 \pm 0.89$ & $41.60 \%$ & $34.10 \%$ & $26.59 \%$ & $19.09 \%$ \\
\hline
\end{tabular}

Table 2: Linear fits of misidentified fraction with respect to distance from the top of the calorimeter: incident energies of $1 \mathrm{TeV}, 10 \mathrm{TeV}, 100 \mathrm{TeV}, 200 \mathrm{TeV}$ and $500 \mathrm{TeV}$. 


\subsection{Charge Detector Segmentation and the Backscattering Effect}

As previously demonstrated [6], a segmented detector is much less prone to back-scatter problems. In the previous section we discussed charge measurement and the backscattering effect for the $2.12 \mathrm{~cm}^{2}$ pixel size segmentation of the ISS-CREAM SCD charge detector. In this section, the backscattering effect with bigger segments is analyzed to demonstrate the segment size dependence on charge resolution. Figure 9 shows the misidentified proton fraction as a function of segment area. The misidentified fraction increases with area, and the area dependence is stronger at higher incident energies. This area dependence increases with energy, as demonstrated in Figure 10, which plots the misidentified fraction vs. area slope as a function of incident energy. For $\mathrm{E}=100 \mathrm{GeV}$ the fraction is $2.4 \%$ with $2.12 \mathrm{~cm}^{2}$ segmentation and increases up to $7.9 \%$ with $766.2 \mathrm{~cm}^{2}$, while for $500 \mathrm{TeV}, 23.5 \%$ and up to $93.3 \%$, respectively. It is clear that fine detector segmentation reduces the effect of back-scatter.

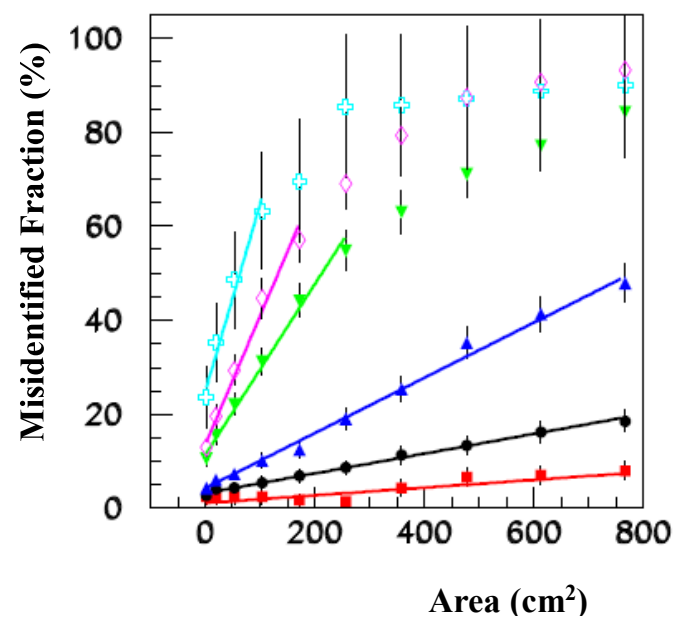

Figure 9: Misidentified fraction over segment size at distance $38.45 \mathrm{~cm}$ : incident energy $100 \mathrm{GeV}$ (red), 1 $\mathrm{TeV}$ (black), $10 \mathrm{TeV}$ (blue), $100 \mathrm{TeV}$ (green), $200 \mathrm{TeV}$ (pink), $500 \mathrm{TeV}$ (light blue), and linear fit (solid line)

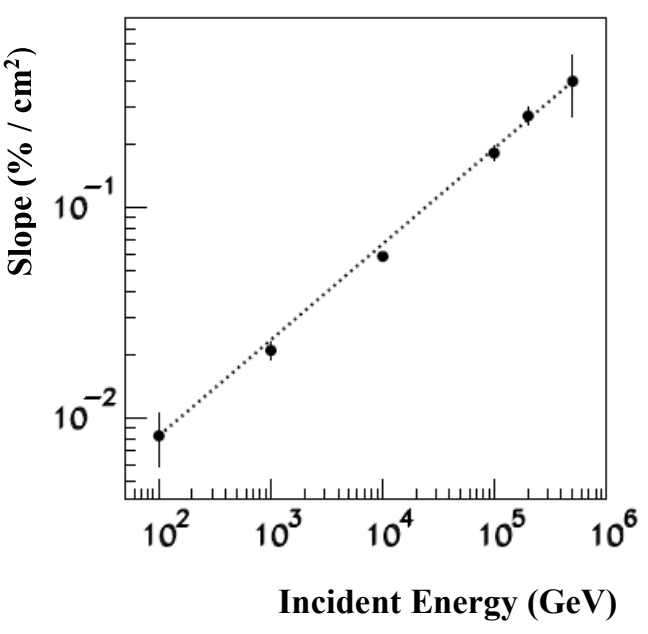

Figure 10: Segment size dependence of misidentified fraction over incident energy. The dotted line is a guide to the eye.

\section{Summary}

It has been shown that the backscattering effect increases with incident energy and increasing detector pixel segment size. The misidentified nuclei fraction in the ISS-CREAM SCD (with $2.12 \mathrm{~cm}^{2}$ segments) is $2.71 \%$ at $1 \mathrm{TeV}$ and increases up to $12.93 \%$ at $200 \mathrm{TeV}$. With a 256.82 $\mathrm{cm}^{2}$ segment size the misidentified fraction is $8.57 \%$ at $1 \mathrm{TeV}$ and increases up to $69.03 \%$ at 200 $\mathrm{TeV}$. For larger segments the energy dependence is even stronger. Our study shows that fine segmentation of the charge detector can limit the backscattering effect quite significantly. Simulations with GEANT4 are currently in progress. The next step will be to compare GEANT3 simulation results with GEANT4. 


\section{Acknowledgements}

This work was supported in the U.S. by NASA grant NNX17AB41G, in Korea by National Research Foundation grants 2018R1A2A1A05022685 and 2018R1A6A1A06024970, and their predecessor grants. It was also supported in France by IN2P3/CNRS and CNES and in Mexico by DGAPA-UNAM project IN109617. The authors thank NASA GSFC WFF and its contractors for engineering support and project management, JSC ISS Program Office for the launch support and ISS accommodation, MSFC for the operational support, and KSC and SpaceX for the launch support. This research was also supported by the Republic of Korea's MSIT (Ministry of Science and ICT), under the High-Potential Individuals Global Training Program (2021-0-01544 and its predecessors) supervised by the IITP (Institute of Information and Communications Technology Planning \& Evaluation).

\section{References}

[1] R. Brun, et al., GEANT User's Guide, CERN, DD/EE/84-1, 1984.

[2] P.A. Arino, et al., FLUKA User's Guide, CERN, TIS-RP-190, 1987.

[3] R.W. Ellsworth, et al., Astrophysics and Space Science, 52, 415-427, 1977.

[4] J. Wu, et al., in proceedings of $36^{\text {th }}$ Int. Cosmic Ray Conf. Pos ( ICRC2019) 154.

[5] J.R. Smith, et al., in proceedings of 35 th Int. Cosmic Ray Conf. Pos (ICRC2017) 199.

[6] E.S. Seo, et al., in proceedings of SPIE 2806, 134-144, 1996.

[7] E.S. Seo, et al., Advances in Space Research, 53/10, 1451-1455, 2014.

[8] Y.S. Yoon, et al., The Astrophys. J., 728, 122, 2011.

\section{Full Authors List: ISS-CREAM Collaboration}

\footnotetext{
S. Aggarwal ${ }^{a, b}$, Y. Amare ${ }^{a}$, D. Angelaszek ${ }^{a, b}$, D. Bowman ${ }^{b}$,Y. C. Chen ${ }^{a, b}$, G. H. Choi ${ }^{c}$, M. Copley ${ }^{a}$, L. Derome ${ }^{d}$, L. Eraud ${ }^{d}$, C. Falana $^{a}$, A. Gerrety ${ }^{a}$, J. H. Han ${ }^{a}$, H. G. Huh ${ }^{a}$, A. Haque ${ }^{a, b}$, Y. S. Hwang ${ }^{e}$, H. J. Hyun ${ }^{e}$, H.B. Jeon ${ }^{e}$, J. A. Jeon ${ }^{c}$, S. Jeong ${ }^{c}$, S. C.

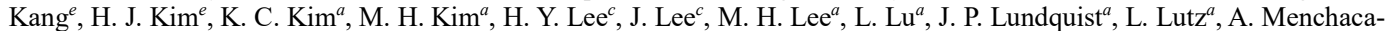
Rocha $^{f}$, O. Ofoha ${ }^{a}$, H. Park ${ }^{e}$, I. H. Park ${ }^{c}$, J. M. Park ${ }^{e}$, N. Picot-Clemente ${ }^{a}$, R. Scrandis ${ }^{a . b}$, E. S. Seo ${ }^{a, b}$, J. R. Smith ${ }^{a}$, R.

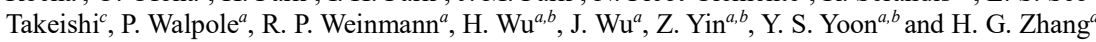

${ }^{a}$ Inst. for Phys. Sci. and Tech., University of Maryland, College Park, MD, USA

${ }^{b}$ Dept. of Physics, University of Maryland, College Park, MD, USA

${ }^{c}$ Dept. of Physics, Sungkyunkwan University, Republic of Korea

${ }^{d}$ Laboratoire de Physique Subatomique et de Cosmologie, Grenoble, France

${ }^{e}$ Dept. of Physics, Kyungpook National University, Republic of Korea

${ }^{f}$ Instituto de Fisica, Universidad Nacional Autonoma de Mexico, Mexico
} 